\title{
Aspects de la réception des classiques dans la Renaissance italienne : le monologue lyrique et la narration épique
}

\section{Marco Praloran}

\section{(2) OpenEdition}

\section{Journals}

Édition électronique

URL : http://journals.openedition.org/edl/391

DOI : $10.4000 /$ edl.391

ISSN : 2296-5084

Éditeur

Université de Lausanne

\section{Édition imprimée}

Date de publication : 15 mai 2010

Pagination : 159-172

ISBN : 978-2-940331-22-2

ISSN : 0014-2026

\section{Référence électronique}

Marco Praloran, «Aspects de la réception des classiques dans la Renaissance italienne : le monologue lyrique et la narration épique », Études de lettres [En ligne], 1-2 | 2010, mis en ligne le 15 mai 2013, consulté le 22 décembre 2020. URL : http://journals.openedition.org/edl/391 ; DOI : https:// doi.org/10.4000/edl.391 


\section{ASPECTS DE LA RÉCEPTION DES CLASSIQUES DANS LA RENAISSANCE ITALIENNE: LE MONOLOGUE LYRIQUE ET LA NARRATION ÉPIQUE}

Un cas fascinant de la reprise du monde classique dans la culture italienne est celui de Pétrarque. Le grand écrivain toscan donna vie à un idéal de renaissance du monde antique qui inaugura l'humanisme: le retour à une langue latine purifiée des sédiments du latin médiéval, la possibilité d'aborder de nouveaux genres se rattachant directement à ceux qui existaient dans l'Antiquité, l'idée même d'une autobiographie idéale, rythmée par une activité épistolaire en latin qui devait promouvoir le rôle nouveau que l'intellectuel européen allait jouer au XVe siècle. Cependant, l'activité littéraire de Pétrarque recèle des aspects moins évidents. Sa production en langue vulgaire surtout, de dimension très réduite par rapport à celle en latin, se concentre sur les thèmes romans de la lyrique amoureuse, dans laquelle le sujet est doté d'une tension tragique tout à fait étrangère à la tradition classique et où la reprise de structures latines dans l'organisation syntaxique elle-même crée des effets d'ambiguïté extraordinaires, signes de l'inquiétude du désir du sujet. Un second aspect est en lien avec le récit chevaleresque $\mathrm{du} \mathrm{XV}^{\mathrm{e}}$ siècle, un genre très éloigné de la tradition épique latine sur le plan des thèmes et de l'intrigue. Toutefois, les auteurs de poèmes chevaleresques italiens, et parmi eux surtout Matteo Maria Boiardo, récupèrent une figure de style caractéristique de l'épopée antique, de l'Enéide, par exemple, ou de la Thébaïde: l'allitération. Les structures allitératives sont employées, comme dans la littérature latine, pour accentuer la tension produite par le récit, pour créer des effets acoustiques qui puissent accompagner la force évocatrice des images, leur impression dans la mémoire.

Il est naturellement impossible de traiter de manière exhaustive de l'évocation du monde classique dans la culture italienne, un aspect très important de la Renaissance. Il est de même impossible de sousestimer le rôle de Pétrarque dans la création de ce que nous appelons communément "humanisme». D’autre part, comme l'a souligné il y a quelques décennies Theodor Mommsen, et comme l'admettaient déjà ses 
contemporains (surtout Boccace), l'idée d'un renouveau influencé par les modèles classiques a été conçue et formulée par Pétrarque.

Au cours de son premier voyage à Rome, Pétrarque fut particulièrement frappé à la vue des ruines et il prit conscience du contraste existant entre un passé resplendissant de grandeur dans toutes ses manifestations et un présent qui le meurtrissait et l'indignait. Comme le rappelle Erwin Panowski, Pétrarque modifia la perception de l'histoire de la pensée chrétienne du Moyen Age ${ }^{1}$; il vit, en réalité, dans son époque une ère de décadence et d'obscurité succédant à l'éclat de la Rome républicaine et impériale. Il vit avec une grande intensité la possibilité d'un renouveau qui pouvait trouver appui, le seul possible, dans la réinsertion des modèles antiques, tant sur le plan culturel et littéraire que civil et politique. Il sentit qu'il était de sa responsabilité d'engager cette transformation. La recherche et le retour à l'étude philologique des textes antiques, une nouvelle conception de la bibliothèque privée (qui retrouvait d'ailleurs l'Antiquité), l'imitation - en ce qui concerne plus précisément la rédaction en latin - des grands modèles de la prose classique, la reprise de genres littéraires depuis longtemps occultés, une conception différente - classique, précisément - de l'écriture privée et de l'écriture publique constituèrent les aspects dominants de ce processus.

Tout ceci est bien connu et a eu un poids considérable dans notre tradition: il n'y aurait pas eu d'humanisme au XVe siècle sans l'enseignement de Pétrarque, sans la construction d'un modèle de pensée et d'action avec comme point de repère le monde intellectuel latin. Et il est curieux, impressionnant même, de découvrir avec quelle attention Pétrarque a construit une autobiographie et une bibliographie pour ainsi dire idéales, en se représentant comme un intellectuel latin moderne. Il est aussi curieux et impressionnant de voir la manière dont il conçut idéalement les étapes de sa vie, de sa carrière d'écrivain et d'homme politique, en forçant les données pour s'inscrire dans la lignée de Cicéron et de Sénèque. La représentation de soi comme intellectuel remonte aux penseurs latins et au rapport articulé mais cohérent entre otium et negotium, entre activité publique et sphère privée. Bien qu'il y ait déjà eu des précédents, ces procédés prennent toute leur importance pour la première fois chez Pétrarque. Il est dès lors possible de dire que le caractère international de sa formation, à Bologne, puis à Avignon auprès de

I. E. Panowski, Renaissance and Renascenses in Western Art. 
la cour papale, son arrière-fond si varié, mêlant culture "vulgaire» italienne, culture classique, connaissance des pères de l'Eglise, philosophie platonicienne et néoplatonicienne (partiellement maîtrisée puisqu'il ne connaissait pas le grec), littérature provençale, etc. - en un mot, cette disposition singulière qui était la sienne, qu'on peut qualifier d'apatride, a permis à Pétrarque de concilier de manière abstraite la représentation de soi comme intellectuel, en adéquation avec les modèles latins. Et ses œuvres, ses succès, ses importantes défaillances (si l'on considère les nombreux projets inachevés) doivent être considérés dans cette optique.

Ce sont là, cependant, des éléments pour le moins connus, et je n'insisterai pas sur ce problème. J'aimerais seulement rappeler que l'œuvre épistolaire de Pétrarque, les Familiares et les Seniles, est essentielle pour reconstruire la vie sociale de l'époque et retrouver les réflexions et les opinions de cet écrivain de génie. On y perçoit également le développement d'une opération d'un certain point de vue artificielle: construire une représentation de soi, un récit autobiographique idéal dominé par un élément fondamental, à savoir le stoïcisme de la culture romaine uni à une sensibilité chrétienne. Les réactions, les indignations et les réflexions de ce Pétrarque sont profondément idéalisées : elles sont pour ainsi dire "antiques", étant donné que l'Antiquité se dévoilait par une intense relecture de sa propre culture littéraire. Ce point de vue particulièrement idéalisé - reconstruit grâce à un processus intellectuel d'assimilation fondé sur une conception entièrement nouvelle de la mémoire empêchant qu'on puisse être oublié avec le temps - apparaît également dans son rapport avec le pouvoir et avec les dirigeants. Dans la lettre qui constitue son testament spirituel, la Posteritati, on perçoit plus que jamais cet objectif: être commémoré comme le fondateur d'un mouvement de renaissance du monde antique. Cette intention est vraiment dominante, même dans les moments les plus chrétiens de l'œuvre de Pétrarque. Par exemple, dans deux fabuleux ouvrages moralisateurs et philosophiques écrits plutôt tardivement - le De otio religioso et le De uita solitaria -, émerge un amour pour la vie frugale et les représentations champêtres qui, bien que contraires à la vie corrompue de la ville, s'unissent au modèle stoïcien de Cicéron et de saint Jérôme. Le style de vie de l'otiosus, empreint de sagesse et de tempérance, l'emporte sur la vie désordonnée des êtres dépourvus de mesure. La solitude que Pétrarque concevait et pratiquait était plus proche de l'otium literatum des antiques 
que de l'ascèse chrétienne: un isolement aristocratique du monde qui permet de se consacrer à la méditation et à la recherche.

Mais tout cela est connu. J'aimerais donc plutôt m'intéresser à certains aspects, peut-être moins communs, de l'imitation des classiques, qui peuvent paraître ambigus et insaisissables. Ces aspects sont particulièrement énigmatiques quand l'imitation ne se développe pas dans la même langue, à savoir en latin, mais d'une langue à l'autre: du latin à la langue "vulgaire». Considérons pour commencer un aspect de nature exclusivement linguistique. On sait que Pétrarque a peu écrit en langue "vulgaire»: sa production latine est beaucoup plus vaste et permet surtout, au moins en apparence, de préciser le profil de l'intellectuel idéal qu'il avait conçu pour lui-même. En réalité, les choses ne se sont pas déroulées comme il l'espérait. Les Vulgarum Rerum Fragmenta ont été le modèle dominant de la poésie européenne durant des siècles, de même que les structures linguistiques et stylistiques, le matériel thématique, le modèle de représentation psychologique du "moi", un modèle absolu, en somme... Bien évidemment, même dans ses œuvres "vulgaires" (qu'il définissait de façon un peu présomptueuse comme des nugae ou nugellae), Pétrarque imite les classiques latins. L'élégance merveilleuse de la syntaxe, la variété et la rareté des figures rhétoriques, la fluidité de l'intonation, la recherche des sonorités, s'apparentent considérablement à la langue des classiques: ils se nourrissent d'elle. Mais il y a plus. Tout le monde sait que l'ordre des mots est particulièrement libre dans la syntaxe latine. C'est la présence des cas qui permet d'appréhender la logique des liens entre les différents éléments. Or ce procédé disparaît dans la syntaxe italienne. En fait, la langue italienne possède une certaine liberté dans l'ordre des mots - liberté certes mesurée, qui complique le décodage des rapports sémantiques. A partir du moment où Pétrarque prend conscience de cette possibilité et la transpose dans la langue poétique "vulgaire", de manière encore plus marquée que ne l'avait déjà fait Dante dans sa Commedia, nous assistons à la création d'effets extraordinaires sur le plan de la configuration et du rythme: le florentin "vulgaire» devient dès lors la langue de la poésie par excellence. Les oscillations rythmiques méticuleuses, et pourtant prodigieuses, la disposition insolite et surprenante, ainsi que les rejets déterminent le plan de la syntaxe, de la rhétorique, du rythme, de la syllabation, comme le préconisent les auteurs antiques dans leurs traités. Mais il ne faut pas oublier que ceci provoque une certaine ambiguïté, qui se répercute sur le lecteur: cette 
ambiguïté reflète les difficultés, les incertitudes du rapport entre le sujet et, d'une part, l'objet de la passion, et de l'autre, le discernement des signes qui lui sont propres. Transposé dans la langue "vulgaire», le classicisme de Pétrarque renferme un aspect sombre, inexistant au départ. Sur le plan de l'imitation, le choix radical de Pétrarque crée de nouvelles possibilités jusqu’alors inexplorées (à l'exception relative de Dante) par la poésie «vulgaire» moderne et accentuent l'éloignement entre la langue poétique et la langue tout court.

Nous avons déjà mentionné le fait que la création d'une biographie intellectuelle est l'une des caractéristiques les plus représentatives de la présence des classiques chez Pétrarque; plus précisément, les rapports entre individu et société sont filtrés et façonnés au travers du modèle des classiques. La langue de cette forme d'activité est le latin, adopté dans les genres les plus diversifiés, de l'épistolographie à l'épopée. Dans l'œuvre latine de Pétrarque se constitue une représentation du "moi» fondée sur le modèle de la philosophie stoïcienne: en somme, il est clair qu'il réussit à faire se rejoindre, notamment au moyen des réflexions augustiniennes, les points de divergence entre la perspective stoïcienne (contrôle rationnel de l'émotion, sentiments filtrés par une humanité touchante et réflexive) et celle de la chrétienté. De là provient l'humanisme italien du $\mathrm{XV}^{\mathrm{e}}$ siècle. Mais à côté du "je» des lettres et des grands traités éthicophilosophiques - à savoir, le De ignorantia, De vita solitaria, etc. - il y a le «je» du Canzoniere et là, les choses changent clairement. Le modèle stoïcien s'étiole peu à peu au moment où il entre en contact avec la tension du désir amoureux. La solution aurait certainement été de concevoir le désir comme un élément marginal de la vie de l'homme. Cependant, Pétrarque superpose à la tradition latine un élément qui n'est pas vraiment classique: il donne pour ainsi dire au thème de l'amour un caractère tragique plutôt qu'élégiaque (même si on perçoit de nombreuses nuances élégiaques dans le Canzoniere). Il s'inscrit ainsi dans la lignée tracée par la lyrique provençale et italienne et surtout par celle, décisive, de Dante, aussi bien dans ses poèmes "petrose", que dans ses théories du De vulgari eloquentia: l'amour devient l'objet de la suprema constructio de la poésie européenne, comme l'a reconnu, il y a environ quarante ans, Erich Auerbach ${ }^{2}$. Le style est sublime, grave, puisque l'élément

2. E. Auerbach, Literatursprache und Publikum in der lateinischen Spätantike und im Mittelalter. 
amoureux a un aspect profondément cognitif: le désir de l'homme est le point de départ d'une réflexion sur l'homme. Ainsi, à la différence de ce qui s'est passé pour le Pétrarque latin, le Canzoniere - où les imitations des poètes latins sont tout de même passablement fréquentes - offre le modèle d'une poésie qu'on ne saurait comparer à celle plus proprement classique, en raison précisément d'une représentation psychologique du «moi» différente. La force existentielle de cette représentation n'est pas comparable. D'autre part, et pour reprendre plus précisément le fil de notre sujet, la pensée, traversée constamment par le désir, ne peut se fixer dans un espace de contrôle et d'équilibre. C'est un discours passionné et non pas impartial, dans le sens stoïcien du terme. Cela présuppose un investissement affectif, un pathos en tous points nouveaux dans la tradition occidentale. Cependant, l'impulsion donnée par la grande tradition moralisatrice latine a tout de même un impact considérable: la variété et la subtilité de la description intérieure sont aussi débitrices de cette culture. C'est pourquoi le «je» lyrique du Canzoniere est infiniment plus modulé et riche que celui de la tradition "vulgaire». Il ne peut être comparé pour l'intensité du réalisme qu'à un autre «je», le "je-narrant» et le «je-narré» de la Commedia. Mais ce qui émerge comme un élément tout à fait nouveau, c'est la variation continuelle des états d'âme, l'impossibilité de poser un filtre rationnel capable de réduire l'angoisse de la passion. Malgré toutes les ambiguïtés de ce discours, celui-ci détient une valeur cognitive presque absolue. Dès lors, le concept de "Renaissance» apparaît certainement comme plus complexe, mais aussi plus intéressant et, dans un certain sens, plus attrayant. De là vient, bien évidemment, l'influence tardive mais immense que l'œuvre de Pétrarque a exercée au cours des siècles successifs, le fait qu'il soit devenu un classique de manière presque analogue à Virgile, en ce sens que son œuvre contient tous les niveaux possibles de l'expression poétique.

Tout en m'excusant de la rapidité et de la fermeté d'un tel discours, j'aimerais à présent diriger mes considérations sur une époque et un genre différents: le poème chevaleresque italien. La tradition et le matériel du poème chevaleresque italien ne sont évidemment pas classiques, mais proviennent bel et bien du Moyen Age. Des trois matières - romane, carolingienne et arthurienne - ce sont les deux dernières qui suscitent le plus d'intérêt auprès du public européen jusque dans la première moitié du $\mathrm{XVI}^{\mathrm{e}}$ siècle. A partir de cette époque, la force normative de la Poétique d'Aristote, enfin traduite en langue «vulgaire» et 
commentée en Italie, annonce le retour des œuvres classiques dans la tradition narrative moderne - retour déjà amorcé grâce à Boccace un siècle et demi auparavant, mais de façon encore peu concluante. La reprise du matériel épique latin - et, à partir d'une certaine époque, du matériel homérique - concerne principalement l'Enéide, mais aussi la Thébaïde de Stace. De façon générale, toutes les œuvres ambiguës quant à leur appartenance générique, telles les Métamorphoses d'Ovide, sont récupérées avec une grande vitalité. A la prolifération du matériel s'ajoute également un élément constructif. La forme et l'articulation de l'intrigue de l'Enéide deviennent le modèle prédominant dès la publication d'un poème peu connu de Gian Battista Trissino, L'Italie libérée des Goths, qui représente la première tentative $\mathrm{d}$ 'imitation du poème latin dans tous ses aspects (même métriques, puisque l'hendécasyllabe privé de rime se substitue à l'octave). Il est évident que la construction polyphonique, fondée sur la pluralité des lignes narratives et typique des poèmes chevaleresques italiens précédents, notamment du Roland furieux de l'Arioste, est aux antipodes du modèle centripète de l'épopée antique. On assiste en réalité à un phénomène des plus intéressants. Le très grand succès du Roland furieux - l'œuvre littéraire la plus diffusée dans l'Italie du XVI ${ }^{\mathrm{e}}$ siècle, plus largement même que le Canzoniere de Pétrarque - incite les exégètes à affilier cette œuvre, de manière presque fébrile, aux classiques antiques et avant tout à l'Enéide, en mettant en évidence tous les critères de l'œuvre qui pourraient évoquer le poème de Virgile: chose facile pour des références ponctuelles (les reprises de Virgile sont très fréquentes dans le Roland furieux), bien plus difficile, voire presque impossible, en ce qui concerne l'intrigue. L'intrigue du Roland furieux est beaucoup plus proche des romans médiévaux, comme le Tristan et le Guiron le courtois, que de l'Enéide. Cependant, l'effort classiciste et l'idée "Renaissance" de modèle, élève cette œuvre au rang de "classique»; plus précisément, l'affiliation au classicisme est due à l'éminence du genre de l'épopée. Lorsque cette opération se révèlera impossible, on recourra alors aux Métamorphoses qui, si elles sont considérées par les traités de l'époque comme un modèle secondaire par rapport au modèle suprême de l'épopée, apparaissent cependant toujours comme un classique incontournable. Dans son projet d'ensemble, le poème de l'Arioste n'a certainement rien à voir avec celui d'Ovide. Or le procédé consistant à réduire à un cas en quelque sorte conforme et acceptable deux ouvres extravagantes devient, dès lors, très significatif. Ce qui est 
toutefois surprenant dans cette opération, c'est l'absence des critères de la tradition romane qui caractérisent indubitablement le Roland furieux. Et cela est non seulement valable pour les romans français en prose du $\mathrm{XIV}^{\mathrm{e}}$ siècle, qui s'éloignent à l'évidence de l'influence "classique", mais aussi pour le Roland amoureux de Matteo Boiardo - œuvre demeurée inachevée quelques décennies avant la composition du Roland furieux (1494) et qui possède d'importants liens avec ce dernier, étant donné que les deux textes s'enchaînent. Ainsi la conversion du Roland furieux en un classique de la littérature italienne moderne se fait au détriment de la connaissance de sa vraie généalogie. Malgré tous les efforts des exégètes, les divergences de l'œuvre par rapport aux modèles classiques - notamment sur le plan de l'intrigue - demeureront un précepte pour certains intellectuels: la dispute entre les partisans de l'Arioste et ses adversaires occupera tout le siècle. Ainsi, quand un nouveau chef d'œuvre apparâ̂tra à la fin du XVIe siècle, à savoir La Jérusalem libérée du Tasse, les adversaires de l'Arioste proclameront la supériorité du Tasse en raison précisément de sa plus grande fidélité aux préceptes aristotéliciens et de la réalisation concrète de l'unité d'action et de temps par rapport à l'Enéide. La nostalgie du retour à la tradition classique (qui domine surtout chez les critiques et dans les manœuvres éditoriales, tandis qu'elle apparaît de façon moins évidente chez le public) ne réussira pas à anéantir l'énorme succès du Roland Furieux de l'Arioste. Elle empêchera en revanche certainement les écrivains postérieurs qui s'essaieront dans le genre épique et même en prose de reprendre son modèle très expérimental en ce qui concerne le développement du temps et des actions des héros. Plusieurs scènes de l'Arioste seront reprises comme nouvelles, même au théâtre, mais simplement comme des épisodes, des morceaux extraits de l'intrigue merveilleuse, et cela non seulement en Italie, mais aussi en Espagne, notamment, où la rencontre du poème de l'Arioste avec la tradition indigène des romans d'aventure en prose donnera naissance au Don Quichotte de Cervantès. D'un certain point de vue - et même si cette observation peut sembler irrévérencieuse - nous pouvons peut-être considérer comme une chance que l'Arioste n'ait pas connu la poétique d'Aristote; sinon, il n'aurait sans doute pas pu concevoir son chef d'œuvre de manière aussi «anticlassique». Ceci mis à part, nous pouvons encore observer que cette petite histoire de la réception met en évidence le concept d'imitation, ainsi que les profondes oscillations que ce concept présuppose dans la Renaissance italienne. 
Mais il est un autre aspect du genre chevaleresque italien que je voudrais souligner. J'ai déjà dit que la tradition littéraire de ces textes est demeurée étrangère au monde classique. Auerbach a bien montré comment les reprises textuelles directes des classiques, par exemple l'épisode du défilé de Camille dans l'Enéide qu'on retrouve dans le Roman de Troie (XII siècle), annihilent le charme et la puissance du récit antique en se perdant dans mille détails secondaires qui, bien que motivés par les événements, rompent le rythme dramatique. Le style sublime du récit épique en hexamètres disparaît dans les octosyllabes de l'ancien français. Quant au mélange du dramatique et du comique, caractéristique des récits d'épisodes guerriers au Moyen Age (comme le prouvent les Chansons de geste), il ne serait pas concevable dans l'épopée latine. Ce critère perdure dans la tradition italienne du $\mathrm{XV}^{\mathrm{e}}$ siècle. Si l'on prend en considération les deux plus grands poèmes chevaleresques - le Morgant de Pulci et l'imposant Roland amoureux de Boiardo que j'ai déjà évoqué à propos du Roland Furieux - nous pouvons observer que, dans les scènes de combats, féroces et impitoyables, les éléments dramatiques sont toujours entrecoupés d'éléments franchement comiques, ce qui a pour effet de provoquer deux sentiments distincts auprès du public: le rire, en raison du caractère hyperbolique des aventures, et la crainte de ce que les héros pourraient sans cesse avoir à subir.

Dans la poésie épique italienne du $\mathrm{XV}^{\mathrm{e}}$ siècle, qui demeure en lien avec la tradition des Chansons de geste lues et diffusées durant plusieurs siècles (composées, même, en Italie, tel le chef d'œuvre l'Entrée d'Espagne qui vit le jour en Italie du nord-est au cours du XIII e siècle), se pose le problème de la représentation des événements. Il existait une tradition populaire, en vogue à partir de la moitié du XIV siècle, qui consistait à «vulgariser» d'importants épisodes de la tradition épique française en couplets de huit vers hendécasyllabiques. La puissance évocatrice du récit épique originairement en "lasse», c'est-à-dire en strophes de longueur variable composées de décasyllabes, laissait la place à un récit plus étendu où l'important était l'intrigue, tandis que la force représentative de la narration l'était beaucoup moins. La construction y est très linéaire, fondée sur une langue passablement conventionnelle et des structures formelles simples, stéréotypées, fortement répétitives: autant de stratégies qui ne trouvaient une réalisation cohérente quau travers de la voix narrative du canterino, du récitant. Il s'agit là d'un genre plutôt bas, dont les reprises du monde classique étaient absentes, ou tout au 
moins épisodiques. Au cours de la deuxième moitié du $\mathrm{XV}^{\mathrm{e}}$ siècle, avec la renaissance de la littérature en langue "vulgaire», ce genre subit une modification. Les intellectuels, fortement influencés par l'humanisme, en prirent possession. Cette littérature est en tous points particulière, encore fortement connotée par une situation qu'un linguiste appellerait pragmatique, c'est-à-dire qu'elle s'ancre dans des circonstances réelles où l'auteur et le public forment une société homogène autour d'une cour aristocratique. C'est le cas du Morgant de Luigi Pulci à Florence et du Roland amoureux de Boiardo à Ferrara. Les deux poèmes démontrent très bien la façon différente dont les deux seigneuries, l'une récente et aristocrate, l'autre antique et féodale, conçoivent le monde de l'héroïsme du Moyen Age, le monde de la chevalerie.

Mais ce qu'il faut surtout souligner, c'est que ces deux écrivains se posent la question de la représentation «figurative» des événements narrés. A la différence de ce qui se passera plus tard au XVIe siècle, la poétique classique n'influence pas le moins du monde le dessein d'ensemble des aventures, ni la forme même de l'intrigue de ces œuvres: elles sont, en somme, privées d'un présupposé théorique. Chez Boiardo, l'intrigue est beaucoup plus expérimentale; c'est une intrigue «romane» et non pas classique, fondée sur la pluralité des lignes narratives, sur une construction polyphonique héritée de la culture française. Dans le Roland amoureux, écrit dans une koinè septentrionale assez différente du florentin littéraire, nous nous trouvons en présence d'une chronologie très complexe, une espèce d'illusionnisme temporel qui rompt avec la solide convention de la narration occidentale, selon laquelle la durée du temps narré doit être objective. Ce choix est probablement lié à l'expérience spatiale caractéristique des arts figuratifs de l'époque. Il n'en demeure pas moins qu'à ce niveau, les rapports avec le monde classique sont inexistants, et l'on peut certainement affirmer que la conception narrative de ce poème est par excellence anticlassique justement parce qu'elle en démantèle les présupposés les plus profonds. Cependant, en dehors du dessein d'ensemble, s'organise une poétique de la représentation de la violence qui se résout plastiquement, figurativement. Nous assistons dès lors à un nouveau regain du monde classique tant sur le plan thématique que, chose peut-être plus surprenante, sur le plan expressif et formel. Sur le plan thématique, ce qui frappe l'imagination de Boiardo, c'est le rude naturalisme de l'épopée antique dans la représentation de la violence: la barbarie, la colère - sur laquelle nous reviendrons - et le 
goût du colossal. Un seul exemple. Dans le troisième livre de l'œuvre, écrit au cours des dernières années du $\mathrm{XV}^{\mathrm{e}}$ siècle, Boiardo narre la terrible bataille qui opposa les païens et les chrétiens sous les murs de Paris. Le héros dominant de cet épisode est un guerrier païen, Rodamonte, qui s'élève au-dessus de la foule des guerriers par son énergie et sa violence. Il est facile de deviner que le modèle de cette représentation s'inspire en grande partie de Capanée, au dixième livre de la Thébaïde de Stace. Le pathos très soutenu, le goût du colossal, le sentiment de terreur qu'il provoque dans une foule sans défense sont des thématiques qui proviennent clairement de Stace; elles sont reprises ici avec une grande fidélité. C’est notamment le cas du passage où Rodamonte émerge des murs de la ville en suscitant la terreur, reprise explicite du passage latin:

Utque petita diu celsus fastigia supra

Eminuit trepidamque adsurgens desuper urbem ${ }^{3}$.

Come egli agionse in cima a quelle mura

E nela tera aparve il gran spavento, levosi un pianto e un strido sì feroce che sino al ciel (credo io) gionse la voce ${ }^{4}$.

Il est très significatif que Boiardo récupère l'image, entièrement empreinte de pathos et plus classique que romane, du grand guerrier immergé dans une foule anonyme et terrorisée. Le dénouement final est tout autant significatif: alors que dans la Thébaïde, il coïncide avec l'éclair lancé par Zeus sur le géant, c'est Roland, qui apparaît ici. Au moment final, au moment décisif, Roland coupe l'échelle de fer au sommet de laquelle se trouve Rodamonte: le guerrier païen tombe dans le fossé, presque entièrement recouvert par les débris de la tour qui s'écroule avec lui. Il s'agit là bel et bien d'une autre reprise de la Thébaïde (XI, 9), mais revisitée par un élément comique absent du modèle classique. L'effet recherché est bien évidemment le rire, comme le suggère la suite du récit, où l'on voit Roland frappé à la tête par un pavé et tombant à terre de façon plutôt ridicule:

E un merlo gionse Orlando nela testa, qual lo distese a terra con tempesta ${ }^{5}$.

3. Thébaïde, X, 870-871.

4. Roland amoureux, III, 8, 29, 5-8.

5. Roland amoureux, III, 8, 31, 7-8. 
La rime riche testa-tempesta nous signale indubitablement l'intention comique. Si Boiardo récupère un modèle représentatif de la tradition classique, les thèmes profondément dramatiques du récit de Stace sont cependant entremêlés d'éléments singuliers, comiques et burlesques, absolument nécessaires dans la tradition italienne et, plus généralement, dans la tradition romane. Haut et bas se retrouvent par conséquent mélangés. Au cours du siècle suivant, plus précisément dans la narration farouchement classiciste du Tasse, tout cela est absolument impossible: il n'y a dans cette œuvre rien qui provoque le rire.

J'aimerais m'attarder sur un dernier élément. Comme nous l'avons souligné, le mouvement et l'animation sont pour Boiardo les caractéristiques essentielles du récit: la bataille et le duel sont le théâtre où ces éléments se réalisent et se concentrent le mieux. Or animation et mouvement représentent un point essentiel de l'art figuratif du XV siècle - qui trouve, entre autres, un modèle fondamental dans la sculpture romane et surtout dans l'art funéraire. Comme cela transparaît dans les traités d'Alberti, les mouvements du corps doivent permettre de lire le mouvement de l'esprit; de même, chez Boiardo, les mouvements de violence deviennent le reflet des mouvements irrationnels et enragés: la colère, l'impétuosité, la rage. Nous savons combien la lecture du De Ira - où Sénèque décrit les effets dévastateurs de cette passion - a été importante au cours du $\mathrm{XV}^{\mathrm{e}}$ siècle italien. Ce dialogue est une œuvre capitale pour saisir la transmission et la formation des codes expressifs. Dans l'Historia, tous les personnages doivent exprimer leurs caractéristiques au travers de la mimique et du geste. Ce sont les formules de pathos de l'art antique qui sont récupérées à cet effet. Dans ce contexte, le caractère figuratif du récit est essentiel, et c'est pourquoi Boiardo agit sur la langue en l'adaptant à un niveau d'expressivité qui lui permet de réaliser ce projet: transmettre et déployer les émotions. L'allitération entre alors en jeu, avec des modalités qui ne sont pas très éloignées de celles présentes dans l'épopée classique. Recourant à l'allitération, Boiardo trouve, de manière presque inattendue, une des multiples possibilités de renouveler certains aspects antiques dans la culture du $\mathrm{XV}^{\mathrm{e}}$ siècle. L'élément spatial obtient ainsi une grande consistance: mouvement et combinaison des corps sont étroitement liés au versant acoustique de la représentation linguistique. On est alors en présence d'un aspect "imitatif» de la langue, en quelque sorte, semblable aux processus de l'épopée antique. Les images sont en partie différentes parce que, chez Boiardo, le style bas s'unit au style 
élevé, et parce qu'il s'éloigne du tragique pur et du thème de la mort des grands guerriers. Cependant, la valeur iconique de la concertation phonique est identique.

J'aimerais conclure sur une image typique, une véritable forme de pathos warburghien: celle du guerrier qui claque des dents sous l'effet de la colère avant d'entrer sur le champ de bataille. En effet, si les héros de Boiardo n'ont pas la même dignité ni la même solennité que les guerriers de Virgile, ils sont tout de même violents:

e sì forte batea dente con dente

dente con dente batte a gran furore

battendo i denti e crollando la testa

l'elmo se allaza con molta tempesta

d'ira soffiando sì come un serpente

mena a doe mano e bate dente a dente

fremendo batte Horilo in forma e denti

più de una arcata se potea sentire

l'un dente contra l'altro screcienire

ma rodendo si va l'ongie col dente

battendo e denti a schiuma come un verro ${ }^{6}$.

L'image se complique progressivement: la fougue des émotions surprend parfois les héros au point de déformer leur corps et d'en vivifier la forme: gestes typiquement dionysiaques, qui évoquent la prodigieuse expérience de Donatello dans la représentation de la violence dans la sculpture italienne au cours de la première moitié du $\mathrm{XV}^{\mathrm{e}}$ siècle.

Marco Praloran

Université de Lausanne

6. Roland amoureux, I, 26, 29, 3; I, 4, 58, 5; I, 26, 7, 7-8 ; I, 28, 24, 7-8, III, 2, 49, 7; I, 15, 33, 7-8; I, 25, 61, 4 et III, 8, 26, 2. 


\section{BIBLIOGRAPHIE}

Textes

Boiardo, Matteo Maria, Inamoramento de Orlando, edizione critica a cura di A. Tissoni Benvenuti e C. Montagnani, Milano/Napoli, 1999.

Stace, Thébaïde, édition J. H. Mozley, London/New York, 1955-1957.

\section{Etudes}

Auerbach, Erich, Literatursprache und Publikum in der lateinischen Spätantike und im Mittelalter, Bern, Francke, 1958.

Panowski, Erwin, Renaissance and Renascences in Western Art, Stockolm, Almquist and Wiksell/Gebers Förlag AB, 1960. 